\title{
Realization of direct bonding of single crystal diamond and Si substrates
}

\author{
Liang Jianbo, Masuya Satoshi, Kasu Makoto, Shigekawa Naoteru
}

\begin{tabular}{|c|l|}
\hline Citation & Applied Physics Letters, 110(11); 111603 \\
\hline Issue Date & $2017-03-16$ \\
\hline Type & Journal Article \\
\hline Textversion & publisher \\
\hline \multirow{3}{*}{ Rights } & $\begin{array}{l}\text { C) } 2017 \text { Author(s). Published by AIP Publishing. } \\
\text { This article may be downloaded for personal use only. Any other use requires prior } \\
\text { permission of the author and AIP Publishing. The following article appeared in } \\
\text { Applied Physics Letters and maybe found at https://doi.org/10.1063/1.4978666 }\end{array}$ \\
\hline DOI & $10.1063 / 1.4978666$ \\
\hline
\end{tabular}

\author{
Self-Archiving by Author(s) \\ Placed on: Osaka City University
}

Liang, J., Masuya, S., Kasu, M., \& Shigekawa, N. (2017). Realization of direct bonding of single crystal diamond and Si substrates. Applied Physics Letters. 110(11); 111603. https://doi.org/10.1063/1.4978666 


\section{Realization of direct bonding of single crystal diamond and Si substrates}

Jianbo Liang, Satoshi Masuya, Makoto Kasu, and Naoteru Shigekawa

Citation: Appl. Phys. Lett. 110, 111603 (2017);

View online: https://doi.org/10.1063/1.4978666

View Table of Contents: http://aip.scitation.org/toc/apl/110/11

Published by the American Institute of Physics

\section{Articles you may be interested in}

Monolithic integration of individually addressable light-emitting diode color pixels

Applied Physics Letters 110, 111103 (2017); 10.1063/1.4978554

Defects responsible for lifetime degradation in electron irradiated n-GaN grown by hydride vapor phase epitaxy Applied Physics Letters 110, 112102 (2017); 10.1063/1.4978641

Fabrication of thin diamond membranes by using hot implantation and ion-cut methods

Applied Physics Letters 110, 101903 (2017); 10.1063/1.4975628

Nature of electron trap states under inversion at $\ln _{0.53} \mathrm{Ga}_{0.47} \mathrm{As}_{/} \mathrm{Al}_{2} \mathrm{O}_{3}$ interfaces

Applied Physics Letters 110, 111602 (2017); 10.1063/1.4977980

Room-temperature direct bonding of silicon and quartz glass wafers

Applied Physics Letters 110, 221602 (2017); 10.1063/1.4985130

Resonance fluorescence of a site-controlled quantum dot realized by the buried-stressor growth technique Applied Physics Letters 110, 111101 (2017); 10.1063/1.4978428

\section{Scilight}

Sharp, quick summaries illuminating the latest physics research






\title{
Realization of direct bonding of single crystal diamond and Si substrates
}

\author{
Jianbo Liang, ${ }^{1, a)}$ Satoshi Masuya, ${ }^{2}$ Makoto Kasu, ${ }^{2}$ and Naoteru Shigekawa ${ }^{1}$ \\ ${ }^{1}$ Electronic Information System, Osaka City University, 3-3-138 Sugimoto, Sumiyoshi, Osaka 558-8585, Japan \\ ${ }^{2}$ Department of Electrical and Electronic Engineering, Saga University, Honjo-machi 1, Saga 840-8502, \\ Japan
}

(Received 10 January 2017; accepted 27 February 2017; published online 16 March 2017)

\begin{abstract}
Diamond/Si junctions have been achieved by surface activated bonding method without any chemical and heating treatments. Bonded interfaces were obtained that were free from voids and mechanical cracks. Observations by using transmission electron microscopy indicated that an amorphous layer with a thickness of $\sim 20 \mathrm{~nm}$ across the bonded interface was formed, and no structural defects were observed at the interface. The amorphous layer of the diamond side was confirmed to be the mixture of $\mathrm{sp}^{2}$ and $\mathrm{sp}^{3}$ carbons by electron energy loss spectroscopy analyzation. The $\mathrm{sp}^{3} /\left(\mathrm{sp}^{2}+\mathrm{sp}^{3}\right)$ ratio estimated from the X-ray photoemission spectra decreased from $53.8 \%$ to $27.5 \%$, while the relative intensity of $\mathrm{sp}^{2}$ increased from $26.8 \%$ to $72.5 \%$ after the irradiation with Ar fast beam which should be predominantly attributable to the diamond-graphite conversion. Published by AIP Publishing.

[http://dx.doi.org/10.1063/1.4978666]
\end{abstract}

Diamond is expected to be the best potential candidate as the next generation semiconductor material for high power and high frequency electronic devices due to its ultimate physical properties, such as the highest electrical breakdown field strength among semiconductors, ${ }^{1}$ high carrier mobility, ${ }^{2}$ and high saturation velocity. ${ }^{3,4}$ Johnson's figure of merit exhibits three times higher $\mathrm{RF}$ power capability than $\mathrm{SiC}^{5}{ }^{5}$ Furthermore, diamond, which possesses the highest thermal conductivity among materials, ten and three times higher than $\mathrm{GaN}$ and $\mathrm{SiC}$, respectively, is the most promising material as a superior heat spreading substrate for power devices.

However, there are several key issues that need to be overcome for the practical application of diamond devices. In particular, monocrystalline diamond substrates are currently limited to a surface area of about $1 \mathrm{~cm}^{2}$. In addition, the cost of the diamond is extremely expensive in comparison with $\mathrm{Si}$. If it is possible to combine single crystal diamond with the large area wafer Si substrate, diamond-based power devices could be fabricated using Si Large Scale Integrated (LSI) process facilities. The combination of diamond devices and Si LSI with various functions on the same substrate is useful for developing electronics application. The direct epitaxial growth of diamond on $\mathrm{Si}$ substrates would be the most desirable approach, but as high as $52 \%$ lattice mismatch and the large difference of the surface energy between $\mathrm{Si}$ and diamond make the growth of diamond on Si substrate more difficult. $^{6-8}$ Therefore, the epitaxial growth of diamond on $\mathrm{Si}$ without interface layers has not actually been realized. As an alternative approach, surface activated bonding (SAB) method could realize the direct combination of two dissimilar materials. ${ }^{9,10}$ We previously applied SAB for making junctions with a variety of dissimilar materials and examined their structural and electrical characteristics. ${ }^{11-13}$

In this work, diamond/Si junctions were fabricated by means of SAB. The structural properties of the bonded interfaces were examined by field emission-scanning electron

\footnotetext{
${ }^{a}$ E-mail: liang@elec.eng.osaka-cu.ac.jp
}

microscopy (FE-SEM), transmission electron microscopy (TEM), and electron energy loss spectroscopy (EELS). Furthermore, the chemical bonding configurations of the diamond substrates without and with Ar irradiation were characterized by X-ray photoemission spectroscopy (XPS). The applicability of the junctions for functional devices was explored based on these measurements.

High-pressure, high-temperature (HPHT) synthetic Ib type (100) single-crystal diamond substrates and $\mathrm{Si}(100)$ substrates were used for our bonding experiments. The sizes of diamond and Si substrates are $4 \mathrm{~mm} \times 4 \mathrm{~mm} \times 0.65 \mathrm{~mm}$ and $12 \mathrm{~mm} \times 10 \mathrm{~mm} \times 0.52 \mathrm{~mm}$, respectively. Prior to the bonding, the average roughness of diamond surface was measured to be $4.08 \mathrm{~nm}$ using atomic force microscope (AFM), which is difficult to band with other substrates. By polishing the surfaces of the diamond substrates, their average roughness was improved from 4.08 to $0.82 \mathrm{~nm}$. Then, $\mathrm{Si}$ and polished diamond substrates were cleaned with acetone and ethanol in an ultrasonic bath for $300 \mathrm{~s}$, dried under $\mathrm{N}_{2}$, and then set in the chamber of SAB facilities. It is noteworthy that prior to bonding, no chemical treatment was utilized on the surfaces of diamond and $\mathrm{Si}$ substrates. Diamond and $\mathrm{Si}$ substrates were bonded to each other at room temperature by $\mathrm{SAB},{ }^{9-12}$ so that diamond/Si junction was fabricated. In the SAB process, we used fast atom beams of Ar to activate both surfaces of $\mathrm{Si}$ and diamond substrates at the same conditions as used previosly. ${ }^{14}$ The chemical bonding structures of the diamond surfaces were evaluated before and after Ar beam irradiation using XPS (ESCA-3400) with a monochromatic $\mathrm{Mg} K \alpha \mathrm{x}$-ray radiation source. The bonding interfaces of the junctions were investigated using FE-SEM (JEOL JSM6500F) and TEM (JEM-ARM200F) equipped with an EELS apparatus. Carbon K-shell edge spectra were taken between 280 and $400 \mathrm{eV}$ at an accelerating voltage of $200 \mathrm{kV}$. The samples for TEM observations were fabricated by a micro-sampling method using a focused ion beam (FIB) technique.

A photograph of diamond/Si bonded sample is shown in Fig. 1(a). A local area of fringe pattern was observed in the 

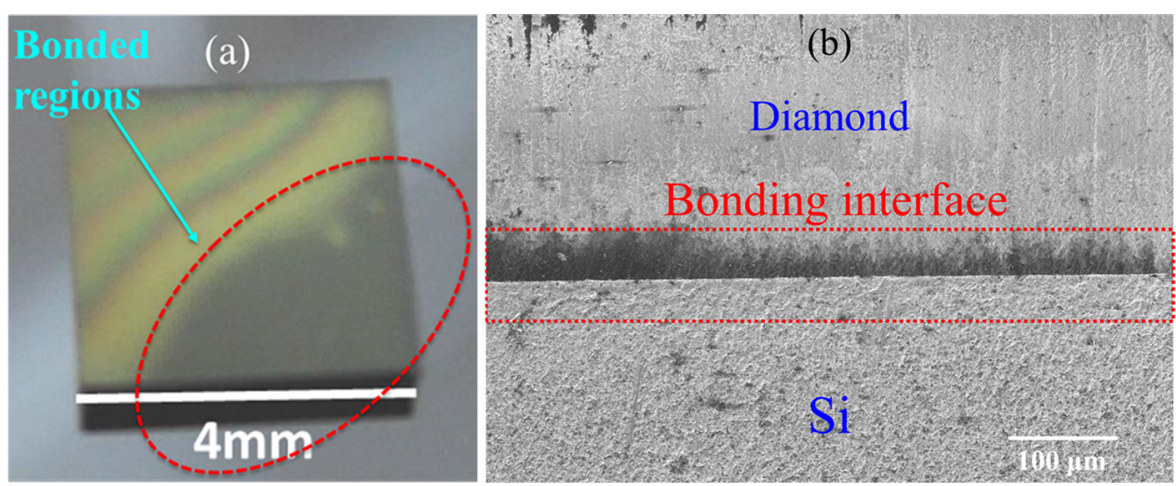

FIG. 1. (a) A photograph of the fabricated diamond/Si junctions sample and (b) FE-SEM cross-sectional image of the bonded interface of diamond/Si.

upper-left part of the sample, which is attributed to the optical absorption by nitrogen impurities in the single crystal diamonds due to the incomplete bonding of diamond and Si. However, no fringe patterns were observed in the bottomright part of the sample, which indicated that the bonded interface of diamond and $\mathrm{Si}$ was achieved. The reason why the full-scale bonding of diamond and $\mathrm{Si}$ could not be obtained is that the polishing produced the uniform thickness of the diamond substrates. The cross-sectional FE-SEM image of the diamond/Si bonded interface is shown in Fig. 1(b). To achieve a smooth cross section of the sample, the mechanical method of polishing is employed to fabricate the SEM sample. The two-layer structure was observed in the sample. The top layer and bottom layer of the sample are diamond and Si, respectively. A straight line can be clearly observed at the center of the sample which corresponds to the interface of bonded substrates. More importantly, no structural deficits or hollow spaces were observed at the interface.

Figure 2(a) shows a TEM cross-sectional image of the diamond/Si bonded interface. There is a transition layer with thickness estimated to be $\sim 20 \mathrm{~nm}$ at the interface, which should be amorphous because it has no lattice fringes and is distinct from adjacent $\mathrm{Si}$ and diamond crystalline phase. Similar to SEM observation, there are no structural defects at the bonded interface. The K-shell edge EELS spectra of two points in the transition layer and a point inside of diamond substrate separated from the interface by approximately $320 \mathrm{~nm}$ are shown in Fig. 2(b). The points for the EELS analyzation are marked point 1 and point 2 in Fig. 2(b), where points 1 and 2 are both inside of the transition layers and point 2 is separated from point 1 by approximately $15 \mathrm{~nm}$. The spectra of points 1 and 2 show a strong $\pi^{*}$ peak at $286 \mathrm{eV}$, which is indicative of $\mathrm{sp}^{2}$ hybridized carbon and is more typical for graphite or amorphous carbon. In addition, the spectral line shape at points 1 and 2 is similar to each other. On the other hand, the spectrum taken at a point inside of the diamond substrates is predominated by the $\sigma^{*}$ peaks at around $292 \mathrm{eV}$, which is typical for the tetrahedral coordination $\mathrm{sp}^{3}$ of carbon in diamond, while a small $\pi^{*}$ peak was observed. This should be related to the damage layer that occurs in the process of the TEM sample fabrication using FIB. The cross-sectional surface of the diamond must be partially transmuted to graphite or amorphous carbon phase after Ar irradiation.

The C1s photoemission spectra of the unirradiated diamond and irradiated diamond substrates are shown in Figs. 3(a) and 3(b), respectively. The fitting of the diamond $\mathrm{C} 1 \mathrm{~s}$ peaks was performed with a combination of Gaussian and Lorentzian functions, after the backgrounds were subtracted using Linear and Shirley's method. As shown in Fig. 3(a), the $\mathrm{C} 1 \mathrm{~s}$ spectrum of the unirradiated diamond was decomposed into three peaks located at $286.2,287.2$, and $288.3 \mathrm{eV}$, which can be assigned to the $\mathrm{C}-\mathrm{C}, \mathrm{C}-\mathrm{O}$, and $\mathrm{C}=\mathrm{O}$ or $\mathrm{O}-\mathrm{C}=\mathrm{O}$ bonding states, respectively. ${ }^{15,16}$ Furthermore, the first and second components correspond to $\mathrm{sp}^{2}$ and $\mathrm{sp}^{3}$ carbon atoms, respectively. By integrating the area of the decomposed peaks, the atomic ratio of $\mathrm{sp}^{3} /\left(\mathrm{sp}^{2}+\mathrm{sp}^{3}\right)$ was estimated to be $53.8 \%$. For the $\mathrm{C} 1 \mathrm{~s}$ spectrum of the irradiated diamond shown in Fig. 3(b), the binding energies of 285 and $286 \mathrm{eV}$


FIG. 2. (a) TEM cross-sectional image of the interface of diamond/Si junction and (b) K-shell edge EELS spectra of the diamond around the interface of diamond/Si junction. 

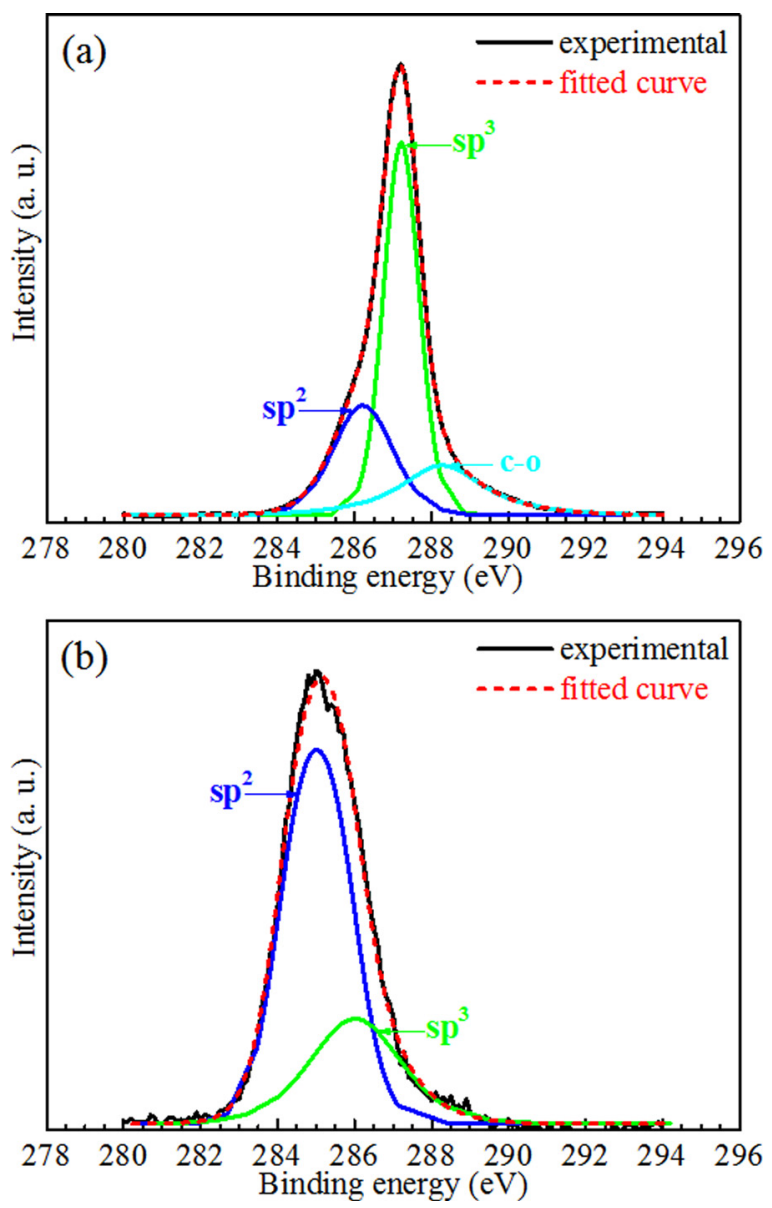

FIG. 3. C1s photoemission spectra of diamond substrates (a) without and (b) with Ar beam irradiation.

were assigned to $\mathrm{sp}^{2}$ and $\mathrm{sp}^{3}$ bonded carbons, respectively. However, the $\mathrm{C} 1 \mathrm{~s}$ bonding energy shifting downward $1.2 \mathrm{eV}$ was observed compared with that of the unirradiated diamond. This should be due to the charging effect and chemical shift. ${ }^{17}$ In addition, the value of $\mathrm{sp}^{3} /\left(\mathrm{sp}^{2}+\mathrm{sp}^{3}\right)$ calculated from the areas of the $\mathrm{sp}^{2}$ and $\mathrm{sp}^{3}$ peaks was approximately $27.5 \%$. The $\mathrm{C}=\mathrm{O}$ or $\mathrm{O}-\mathrm{C}=\mathrm{O}$ peak originated from the substrate surface disappeared after Ar fast beam irradiation. A similar result was observed in polycrystalline diamond, in which the $\mathrm{C}-\mathrm{O}$ or $\mathrm{C}-\mathrm{O}-\mathrm{C}$ and $\mathrm{C}=\mathrm{O}$ or $\mathrm{COOH}$ peaks were removed by ion bombardment etching. ${ }^{15}$

The $\mathrm{sp}^{3}$ peak gradually broadened and weakened after Ar irradiation. In other words, the $\mathrm{sp}^{2}$ peak strengthened with $\mathrm{Ar}$ irradiation. This phenomenon might predominantly be due to a few parts of the diamond crystallites that were decomposed to amorphous carbon or graphite. It has been reported that the $\mathrm{sp}^{3}$ bonding fraction of ultra-nanocrystalline diamond was reduced due to hydrogenated amorphous carbon. ${ }^{18}$ In addition, the full width at half maximum (FWHM) of the $\mathrm{sp}^{3}$ peaks were calculated to be 1.1 and $2.75 \mathrm{eV}$ for the unirradiated and irradiated diamond, respectively. The FWHM value for the irradiated diamond is significantly larger than that of the unirradiated diamond. Furthermore, the $\mathrm{sp}^{3} /\left(\mathrm{sp}^{2}+\mathrm{sp}^{3}\right)$ value of the irradiated diamond is much smaller than that of the unirradiated diamond. These results indicate that the presence of a large number of graphite or amorphous carbons on the substrate surfaces degrades the value of $\mathrm{sp}^{3}$. The difference in the FWHM of the $\mathrm{sp}^{3}$ peaks is because the wave function around the Fermi level is disordered in amorphous or graphite carbons rather than in a crystalline diamond structure. ${ }^{19}$ The detachment of the localized electron states from the valence and the conduction bands occurs in amorphous or graphite carbons, which would lead to a change in the density of available states. This change is reflected in the interlayer distance of the disordered carbons.

On the basis of the experimental results, we found that the amorphous layer across the bonded interface consists of the amorphous layers of $\mathrm{Si}$ and diamond sides; the amorphous layer thickness of the diamond side was estimated to be $\sim 15 \mathrm{~nm}$ and the amorphous layer of the diamond side was determined to be amorphous or graphite carbons by EELS analyzation. Although the thickness of the amorphous layer of the diamond side is markedly thicker than that of the $\mathrm{Si}$ side, the thickness of the Si side is consistent with our previously reported result on $\mathrm{Si} / \mathrm{Si}$ junctions fabricated by $\mathrm{SAB} .{ }^{14}$ The main reason should be related to the feature of the bonding materials and would need to be investigated in the future. The $\mathrm{sp}^{3} /\left(\mathrm{sp}^{2}+\mathrm{sp}^{3}\right)$ ratio estimated from the XPS decreased from $53.8 \%$ to $27.5 \%$ with the irradiation of $\mathrm{Ar}$ fast beam, which contributed to the decrease of the ratio of the diamond and the increase of the portion of the amorphos carbon or graphite originating from the collapse of the diamond cyrstal structure. A similar phenomenon at the SAB-based bonded interface was also observed in the $\mathrm{Si} / \mathrm{Si},{ }^{19,20} \mathrm{Si} / \mathrm{SiC},{ }^{12}$ and $\mathrm{GaP} / \mathrm{GaAs}^{21}$ junctions. The importance of the existence of amorphous layer across the bonded interface is unavoidable for realizing the bonding of $\mathrm{Si}$ and diamond without intermediate layer. The main reason is that the amorphous layer could release the stress caused by the difference in lattice constants and thermal expansion coefficient between Si and diamond. No structural defects were observed at the interface, which indicated that $\mathrm{Si}$ and diamond were firmly bonded to each other. If a large number of small size single crystal diamonds could be regularly embedded in a large area $\mathrm{Si}$ substrate, which could be as a large area diamond substrate to use. Furthermore, the embedded diamond substrate is very useful for large area crystal growth of diamond. For instance several diamonds could be embedded in the $\mathrm{Si}$ substrate, which are oriented in the same direction and coherent with each other. The embedded diamonds would be used as three-dimensional seed crystals. And then a homoepitaxial layer could be grown on the tops of the diamonds and connected with all of the diamonds. Finally, the largediameter diamond substrate would be obtained. Moreover, it is expected that diamond/Si junctions accelerate the research of diamond materials and devices. These results suggest that the SAB-based diamond/Si junctions are anticipated to play a significant role in fabricating devices operating for high-power and high-frequency applications.

In conclusion, we fabricated diamond/Si junctions by using surface-activated bonding without any treatment and intermediate layer. No voids and mechanical cracks were observed at the bonded interface. An amorphous layer was observed at the interface, which was due to the Ar fast beam irradiation induced damage layer. EELS measurements revealed that the contributions of $\pi^{*}$ and $\sigma^{*}$ orbitals were observed in the vicinities of bonded interfaces and the amorphous layer was composed of $\mathrm{sp}^{2}$ and $\mathrm{sp}^{3}$ carbons. Both $\mathrm{sp}^{2}$ 
and $\mathrm{sp}^{3}$ signals of the carbon atoms were observed on the diamond surfaces by XPS measurements. The value of the $\mathrm{sp}^{3} /\left(\mathrm{sp}^{2}+\mathrm{sp}^{3}\right)$ ratio decreased from $53.8 \%$ to $27.5 \%$ with $\mathrm{Ar}$ irradiation.

This work was supported by the Grant-in-Aid for Challenging Exploratory Research (16K13676) of the Ministry of Education, Culture, Sports, Science, and Technology (MEXT), Japan.

${ }^{1}$ A. T. Collins, in Properties and Growth of Diamond, edited by G. Davies (Inspec, London, 1994).

${ }^{2}$ J. Isberg, J. Hammersberg, E. Johansson, T. Wikström, D. J. Twitchen, A. J. Whitehead, S. E. Coe, and G. A. Scarsbrook, Science 297, 1670 (2002).

${ }^{3}$ F. Nava, C. Canali, C. Jacoboni, L. Reggiani, and S. F. Kozlov, Solid State Commun. 33, 475 (1980).

${ }^{4}$ L. Reggiani, S. Bosi, C. Canali, F. Nava, and S. F. Kozlow, Phys. Rev. B 23, 3050 (1981).

${ }^{5}$ E. Kohn and W. Ebert, in Low-Pressure Synthetic Diamond, edited by B. Dishcler and C. Wild (Springer, Berlin, 1998).

${ }^{6}$ K. Ishibashi and S. Furukawa, Jpn. J. Appl. Phys. 24, 912 (1985).

${ }^{7}$ X. Jiang, C.-P. Klages, R. Zachai, M. Hartweg, and H.-J. Fusser, Diamond Relat. Mater. 2, 407 (1993)
${ }^{8}$ C. J. Tang, A. J. Neves, and A. J. S. Fernandes, Diamond Relat. Mater. 12, 251 (2003).

${ }^{9}$ H. Takagi, K. Kikuchi, R. Maeda, T. R. Chung, and T. Suga, Appl. Phys. Lett. 68, 2222 (1996).

${ }^{10}$ C. Wang, E. Higurashi, and T. Suga, Jpn. J. Appl. Phys. 47, 2526 (2008).

${ }^{11}$ J. Liang, T. Miyazaki, M. Morimoto, S. Nishida, N. Watanabe, and N. Shigekawa, Appl. Phys. Express 6, 021801 (2013).

${ }^{12}$ J. Liang, S. Nishida, M. Arai, and N. Shigekawa, Appl. Phys. Lett. 104, 161604 (2014).

${ }^{13}$ J. Liang, S. Nishida, M. Morimoto, and N. Shigekawa, Electron. Lett. 49, 830 (2013).

${ }^{14}$ J. Liang, T. Miyazaki, M. Morimoto, S. Nishida, and N. Shigekawa, J. Appl. Phys. 114, 183703 (2013).

${ }^{15}$ D. Bullutaud, N. Simon, H. Girard, E. Rzepka, and B. Bouchet-Fabre, Diamond Relat. Mater. 15, 716 (2006).

${ }^{16}$ B. Humbert, N. Hellala, J. J. Ehrhardt, S. Barrat, and E. Bauer-grosse, Appl. Surf. Sci. 254, 6400 (2008).

${ }^{17}$ C. D. Wagner, J. Electron Spectrosc. Relat. Phenom. 10, 305 (1977).

${ }^{18}$ S. Adhikari, D. C. Ghimire, H. R. Aryal, S. Adhikary, H. Uchida, and M. Umeno, Diamond Relat. Mater. 15, 1909 (2006).

${ }^{19}$ T. T. P. Cheung, J. Appl. Phys. 53, 6857 (1982).

${ }^{20}$ H. Takagi, R. Maeda, N. Hosoda, and T. Suga, Jpn. J. Appl. Phys. 38, 1589 (1999).

${ }^{21}$ M. M. R. Howlader, T. Suga, F. Zhang, T. H. Lee, and M. J. Kim, Electrochem. Solid-State Lett. 13, H61 (2010). 